\title{
Démarche Estratégica: modo inovador e eficaz de análise da missão institucional
}

\author{
| ${ }^{1}$ Maria de Jesus Dias de Araújo, ${ }^{2}$ Elizabeth Artmann, \\ ${ }^{3}$ Maria Angélica C. Andrade I
}

Resumo: No Brasil, apesar das melhorias ocorridas na saúde das mães e crianças, os indicadores de mortalidade materna e neonatal ainda estão distantes do desejável. Para a reversão desse quadro, é importante repensar os processos de trabalho, considerando o ambiente hospitalar e a rede de cuidados perinatais do território, e apostar na democratização institucional. O objetivo deste estudo foi discutir o processo de aplicação do enfoque "démarche estratégica” numa maternidade púbica estadual, numa perspectiva de rede coordenada de serviços. O período foi de junho a setembro de 2009 e incluiu a participação direta dos profissionais/atores envolvidos, inclusive a direção do hospital. Os resultados mostraram a capacidade do método para problematizar os processos de trabalho e para a análise da gestão do hospital. A experiência permitiu, a partir das análises, apontar estratégias de ação validadas pelos diferentes atores. $\mathrm{O}$ método pode ser considerado um modo inovador e efetivo para produzir equipes de saúde mais solidárias e corresponsáveis com movimentos de mudança das práticas. Permitiu a criação de canais de comunicação entre os profissionais da maternidade, e entre estes com atores da rede externa. $\mathrm{O}$ estudo mostrou a potencialidade do enfoque como um instrumento de diagnóstico situacional e de ferramenta de gestão hospitalar para rever a missão institucional na busca de qualidade e solidariedade dentro da rede e no compromisso com o atendimento ao usuário de forma universal e equânime. Contudo, os compromissos expressos na síntese propositiva devem ser constantemente revistos e repactuados entre os atores, para alcançar mudanças institucionais permanentes.

1 Mestrado em Gestão do Trabalho e Educação em Saúde EP/Fiocruz. Supervisora Teda SESAPI - Piauí, Teresina. Endereço eletrônico: jesusdiasf@gmail.com

${ }^{2}$ Doutora em Saúde Coletiva pela UNICAMP; pesquisadora titular da Escola Nacional de Saúde Pública/FIOCRUZ. Endereço eletrônico: artmann@ ensp.fiocruz.br

${ }^{3}$ Médica no Hospital Dorio Silva/Serra e professora adjunta do Departamento de Medicina Social da UFES. Endereço hotmail.com 


\section{Introdução}

A saúde materna e perinatal é de grande relevância, pelo fato de as mulheres e seus recém-nascidos representarem um grupo com alta vulnerabilidade, expressa na maior exposição ao risco de adoecer e morrer. Mais de 500.000 mulheres morrem no mundo inteiro todos os anos devido a complicaçóes relacionadas à maternidade (DAVIS-FLOYD et al., 2010), configurando uma crise na saúde materna e infantil.

No Brasil, nas últimas décadas, mesmo com as melhorias ocorridas na saúde das mães e crianças, os indicadores de mortalidade materna e neonatal ainda estão distantes do que se consideraria aceitável e desejável (PASCHE et al., 2010). Apesar de as estimativas das razões de mortalidade no Brasil serem afetadas pelo sub-registro de óbitos, as mortes maternas ainda são muito elevadas e consideradas uma violação dos direitos reprodutivos das mulheres, visto que são evitáveis com atenção à saúde precoce e de qualidade.

Os coeficientes de mortalidade infantil decresceram acentuadamente nos últimos trinta anos, mas foram acompanhados por reduções menores nos óbitos neonatais e pelo aumento da ocorrência de nascimentos prematuros (VICTORA et al., 2011). Nos últimos vinte anos, melhoraram quase todos os outros indicadores da saúde materna e das mulheres, assim como o acesso aos serviços de saúde (DINIZ, 2009). Agrega-se a esta constatação o fato de a assistência ao parto no Brasil ser essencialmente institucionalizada, ou seja, realizada principalmente em hospitais e por profissionais de saúde habilitados (médicos, em sua maioria). Este fenômeno complexo e desafiador, denominado "paradoxo perinatal", revela que o maior acesso à tecnologia, aos insumos e equipamentos biomédicos, maior presença de profissionais e formulações normativas não garantem, por si só, a reversão em tempo esperado do quadro de mortalidade materno-infantil (PASCHE et al., 2010).

Para a reversão desse quadro, impõe-se repensar a organização dos processos de trabalho, tendo por alvo o ambiente hospitalar e a rede de cuidados perinatais do território onde se encontra inserida a maternidade e apostar na democratização institucional. Segundo Pasche, Vilela e Martins (2010), esse processo se depara com a paradoxal realidade dos hospitais brasileiros, que são responsáveis por avanços e pela qualificação no cuidado em saúde, mas, ao mesmo tempo, estão entre as instituições contemporâneas que resistem às mudanças, às inovações, sobretudo na gestão. 
Para que esta aposta demande "um reposicionamento dos sujeitos na experiência concreta de produção da realidade" (PASCHE et al, 2010, p. 569), ela deve produzir consensos suficientes para desencadear processos de decisão institucional e, consequentemente, opçôes estratégicas para as mudanças necessárias (BENEVIDES; PASSOS, 2005). Nesse sentido, reconhecer a primazia comunicacional supóe tomar como objeto de análise a competência comunicativa entre os sujeitos trabalhadores, gestores e usuários dos serviços de saúde e priorizar estratégias relacionais e de aliança capazes de superar os desafios, a fim de assegurar melhores práticas de cuidado.

Para enfrentar essas dificuldades e desafios, segundo Cecílio (1997), é necessária a emergência de novos modelos de gestão para a efetivação de mudanças nas práticas de saúde, pois, mesmo sendo utilizada uma série de tecnologias de planejamento e gestão, a maioria dos modelos adotados, sobretudo pelos hospitais públicos, pouco tem contribuído para enfrentar estas questôes.

A promoção de inovações nos processos e instrumentos de gestão visa alcançar maior efetividade, eficiência e qualidade de suas respostas. Esse processo de planejamento deve ser desenvolvido de forma articulada, integrada e solidária, com base nas três esferas de gestão, pressupondo que cada qual realize seu planejamento, articulando-se de forma a fortalecer e consolidar os objetivos do SUS (BRASIL, 2006).

Vivemos uma crise de governabilidade do sistema e das organizações de saúde, de resolutividade e eficiência. Segundo Crémadez e Grateau (1997), a erosão de certos paradigmas tradicionais (formação e processo de trabalho médico, do serviço público, profissional de saúde) estaria na origem da uma crise de identidade organizacional que enfraquece o contrato que liga os indivíduos à organização. $\mathrm{O}$ resultado do impacto dessas mudanças aponta para o surgimento de características sócio-culturais incoerentes com o padrão tradicional, identificando-se hoje, um quadro cultural contraditório, em transição (RIVERA, 2003). Além disso, esta crise de identidade gerada por essas mudanças acentua sentimentos e reações negativas, principalmente entre o grupo de profissionais médicos, que, como grupo dominante, contesta as políticas e tentativas de racionalização gerencial. No plano externo, o hospital torna-se mais reativo, superestima restriçōes de recursos e passa a olhar o futuro com inquietação. Faz parte do pano de fundo dessa crise a necessidade de fortalecer a regulação e de introduzir critérios de 
gestão nos centros operacionais, o que ameaça muitas vezes a autonomia dos profissionais, especialmente médicos (CRÉMADEZ; GRATEAU, 1997).

É nesse contexto que a démarche estratégica propóe-se a ensejar movimentos que permitam a construção de projetos comuns, fortalecendo a comunicação entre direção e ponta, e entre os diversos serviços. Mais especificamente, os objetivos da démarche seriam: promover um elo entre a gerência estratégica e os centros assistenciais, através da comunicação; definir a missão do hospital de acordo com o critério de oportunidade, o qual equivale a determinar um padrão de atividades que lhe permita vantagens comparativas; ensejar um lugar de comunicação, onde os atores-serviços possam chegar a definir um projeto coletivo; favorecer a integração do hospital em uma rede de oferta de cuidados estruturada com a preocupação da eficácia e da eficiência; promover um processo de transformação cultural progressiva, não necessariamente preso à busca de objetivos instrumentais, imediatos (RIVERA, 1997).

$\mathrm{Na}$ perspectiva político-organizacional, o enfoque se opõe a uma definição normativa e tecnocrática de diretrizes, apostando na via da responsabilização dos atores que implica o desenvolvimento de processos comunicativos e de negociação internos e externos, que redundem em acordos relativos aos projetos assistenciais pertinentes a uma rede (ARTMANN; RIVERA, 2003). A maternidade escolhida como objeto deste estudo traduz esta demanda, apontando no sentido de um estudo de avaliação de sua organização.

$\mathrm{O}$ objetivo deste estudo foi descrever o processo de aplicação do enfoque “démarche estratégica” numa maternidade púbica estadual, utilizado como método de análise da gestão e como avaliação da instituição hospitalar numa perspectiva de rede coordenada de serviços.

\section{Método}

O estudo foi realizado no período de junho a setembro de 2009 em uma maternidade pública estadual, localizada na região sul da cidade de Teresina, Estado do Piauí, escolhida por sua localização estratégica, por ser referência estadual e regional e por ser um hospital de ensino. Foi inaugurada em 1976, com 240 leitos obstétricos, como a única maternidade pública da capital do estado. Nos seus 33 anos de existência, tem passado por reformas estruturais, sendo que, 
atualmente, possui 248 leitos obstétricos, dos quais 177 leitos neonatais. É a maior maternidade do estado e responsável por $63 \%$ dos nascimentos ocorridos na cidade de Teresina. Apresenta, em média, 1.400 internações por mês, dentre as quais, 1.364 partos mensais. Destes partos, apenas cem são realizados por convênios e ou particulares, 664 são partos normais e 600 são partos cesarianos e outras intervençōes cirúrgicas (SESAPI, 2009).

Com a implantação da Gestão Plena Municipal no Sistema Único de Saúde (SUS), a maternidade passou a ser referência também na rede municipal, no tocante à assistência à gestante de alto risco. Ela é campo de estágio dos cursos de graduação e pós-graduação das universidades federal e estadual (medicina, enfermagem, nutrição, serviço social, odontologia, fisioterapia e enfermagem). Possui os programas de residência médica em obstetrícia/ginecologia e de pediatria (área de concentração: neonatalogia), credenciados pela Comissão Nacional de Residência Médica.

A pesquisa teve início após autorização do Comitê de Ética em Pesquisa da Escola Nacional de Saúde Pública Sérgio Arouca, sob o Protocolo de Pesquisa CEP/ ENSP no 55/09, CAAE: 0075.0.031.000-09, e autorização do Comitê de Ética em Pesquisa da maternidade em estudo, sob protocolo no 667/04. Foi assinado o termo de consentimento livre e esclarecido para participação na pesquisa, segundo Resolução CNS no 196/96, pela direção geral e pelos profissionais envolvidos. Da primeira reunião e a reunião ampliada final, participou o diretor da maternidade. O enfoque "démarche estratégica" foi aplicado com participação direta dos atores/profissionais: diretor do Instituto de Perinatologia/médico; diretor técnico/médico; diretor financeiro/administrador; coordenadora da neonatologia; coordenador do comitê de ética; coordenador de ensino e pesquisa; coordenadora de enfermagem; coordenadora da nutrição e responsável pelo banco de leite/nutricionista; responsável pelo centro obstétrico; coordenador da Câmara Técnica de Humanização e cinco voluntários; três assistentes sociais, um responsável pela ouvidoria e um administrador. Ao todo foram 19 reuniōes, com média de sete participantes em cada, considerando as seguintes fases:

a) Análise do existente: corresponde ao diagnóstico administrativo e médico; inclui a lista de parceiros e concorrentes e a rede de cuidados. O diagnóstico administrativo refere-se às informaçōes tradicionais, tais como o tempo médio 
de permanência, o número de ingressos, o pessoal médico e paramédico, demanda e estrutura física, etc. $\mathrm{O}$ diagnóstico médico compreende a relação de patologias atendidas por especialidades médicas, de acordo com a complexidade; a relação de tecnologias utilizadas por especialidades, de acordo com a complexidade; o cruzamento entre patologias e tecnologias, identificando a incidência das tecnologias sobre as patologias; a relação das modalidades de atenção, indicando se as atividades são programáveis ou não e se a intervenção demanda consulta ambulatorial e/ou hospitalização; o tipo de hospitalização e o tempo da internação requerida, apontando para a necessidade de um tipo de serviço existente no na unidade de saúde ou fora dela.

b) Segmentação: definição dos principais agrupamentos homogêneos de atividades, geralmente por especialidades, considerando as categorias "patologia", "tecnologia", "modos de atenção" e "população", que permitam uma análise estratégica representativa da instituição.

c) Análise do valor do segmento: refere-se ao nível de prioridade relativo que os segmentos teriam para a especialidade e se expressa pelo interesse em lhes alocar recursos. O objetivo central é a valorização dos segmentos por meio de uma ponderação de critérios e de uma nota relativa. Cada critério recebeu determinado peso em termos percentuais na fixação do valor do segmento; este peso é variável e é referente à especialidade. Atribui-se, por consenso, uma nota de 0 (zero) a 20 (vinte) por critério. Foram considerados na matriz para análise de valor os seguintes critérios: 1) possibilidade de crescimento; 2) intensidade da concorrência; 3) investimento em equipamento e tecnologia (barreira à entrada); 4) investimento em RH (barreira à entrada); 5) sinergias; 6) possibilidade de parceria externa; e 7) potencial de ensino e pesquisa. A possibilidade de crescimento refere-se às perspectivas de desenvolvimento. Leva-se em consideração a evolução das técnicas de diagnóstico e de terapia, considerando-se o cenário epidemiológico-social e o tecnológico. A intensidade da concorrência refere-se à previsão do quantitativo de instituições e de profissionais que realizam a mesma atividade. As barreiras de entrada relacionam-se ao montante do investimento envolvido no desenvolvimento do segmento. Este investimento pode ser desdobrado em equipamentos e tecnologia, capacidade instalada, recursos humanos especializados. As 
sinergias referem-se à possibilidade de compartilhamento de equipamentos, tecnologias, capacidade instalada, apoio logístico e opiniāo dos profissionais. As possibilidades de parcerias externas dizem respeito ao potencial de captação de recursos externos por meio do desenvolvimento de cada segmento, de melhorar a imagem externa da especialidade e do hospital. As potencialidades dos segmentos correspondem à capacidade de atração de clientela de nível regional e local e às capacidades de geração de atividades de ensino e de pesquisa. Nessa fase, o resultado é uma descrição precisa dos problemas específicos do segmento.

d) Análise da Posição Competitiva e Fatores-Chave de Sucesso: considera-se o grau de controle sobre os Fatores-Chave de Sucesso (FCS), que correspondem a vantagens para a garantia de êxito em uma atividade. A identificação dos FCS é feita pelo levantamento dos concorrentes; ponderação dos FCS, que consiste em distribuir cem pontos entre os FCS em função de sua importância relativa; notação, entendida como o ato de distribuir nota de 0 a 20 a cada FCS em função do grau de controle real dos mesmos, considerando o controle próprio e o controle dos concorrentes/parceiros; determinação da posição relativa própria e dos concorrentes, por meio da comparação dos escores pertinentes, sendo o escore de cada concorrente determinado a partir do produto do peso e da nota por FCS.

e) Portfólio de atividades: constitui-se a partir do valor e posição competitiva e do volume de produção dos segmentos, permitindo determinar prioridades e desenhar estratégias gerais que podem ser de crescimento, manutenção ou diminuição das atividades, ou ainda, de criação de um segmento. Representa um instrumento que permite a visualização imediata do desempenho da instituição analisada através de um gráfico constituído pelo valor como ordenada e abscissa como posição competitiva. Cada segmento é representado por um círculo, cujo tamanho representa o volume de produção das atividades que ele representa, e sua posição depende do escore obtido quando foi feita a avaliação do valor e do grau de controle dos FCS, o que define sua posição competitiva. Contribui para determinar prioridades e para fixar as estratégias de ação. 


\section{Resultados e Discussão}

A aplicação da démarche foi iniciada a partir de reuniōes com profissionais do serviço de atendimento médico e estatística (SAME), faturamento, arquivo e sistema de informação da maternidade para levantamento das informações, dados de produção, dados epidemiológicos, demanda e oferta. Nesse processo, destaca-se a descoberta da ausência de faturamento de uma das quatro alas de alojamento pertencente à Unidade Materno-Infantil. Esse fato foi observado a partir da análise da produção no Sistema de Informações Hospitalares (SIH), baseado nas Autorizações de Internação Hospitalar (AIH).

Posteriormente, realizaram-se reuniōes semanais na maternidade com os profissionais médicos e de enfermagem da Unidade Materno-Infantil, com participação direta das chefias. Destaca-se a importância desta participação no processo de discussão, por motivar a participação e o diálogo entre os profissionais dos setores e a direção estratégica, integrando diferentes serviços operacionais. É importante observar que a dinâmica da aplicação do enfoque utilizou formulários específicos e muitas discussōes, "idas e vindas" entre os profissionais da maternidade para reavaliação das notas atribuídas, após comparação e verificação de dados e argumentos em que se baseou a notação.

Realizou-se levantamento de todas as patologias atendidas e dos procedimentos realizados pela Unidade Materno-Infantil da maternidade e, posteriormente, foram definidos os segmentos mais ou menos homogêneos de atividades, agrupados de acordo com os critérios "tecnologia", "população", "modo de atenção" e "patologia": UTI Neonatal, UCI Neonatal, Alojamento Conjunto de Risco, Obstetrícia, Imunização, Pré-natal, Apoio Diagnóstico e Imunização. Ressaltase que a definição dos segmentos é um processo dinâmico que inclui a clareza dos critérios e a explicitação das motivações envolvidas. Além disso, embora os critérios façam parte intrínseca do enfoque, é importante que sejam agregados dados e informações de caráter mais objetivo nessa escolha (ARTMANN, 2002).

$\mathrm{Na}$ fase de valorização dos segmentos, foram atribuídos os valores percentuais aos critérios previstos no método (tabela 1). A distribuição percentual dos pesos refletiu a valoração e hierarquização dos critérios por 
parte do grupo de profissionais envolvidos. Os maiores pesos contamplaram os critérios "possibilidade de crescimento" e "potencial de ensino e pesquisa", tendo em vista a recente ampliação de leitos da UTI neonatal e o fato de a maternidade ser campo de estágio para duas universidades públicas, duas faculdades privadas, e para os estudantes de nível técnico. Os menores pesos foram atribuídos à intensidade da concorrência, investimento em recursos humanos e possibilidade de parceria externa (a ser implementada inclusive com outros hospitais da rede) e por considerar a necessidade de contratação de especialistas, tais como neuropediatra e cardiopediatra.

Tabela 1. Valor dos segmentos da unidade materno infantil de uma maternidade pública estadual, segundo critérios e peso de avaliação. Teresina-PI, 2009

\begin{tabular}{|c|c|c|c|c|c|c|c|c|}
\hline \multirow[b]{2}{*}{ Critérios de Avaliação } & \multicolumn{7}{|c|}{ NOTA } & \multirow[b]{2}{*}{ 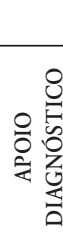 } \\
\hline & \begin{tabular}{l}
$\circ$ \\
0 \\
0 \\
0 \\
\cline { 1 - 1 }
\end{tabular} & 巨。 & تี & 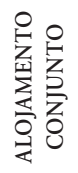 & 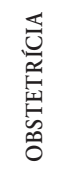 & 岕 & 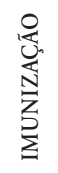 & \\
\hline Possibilidade de crescimento & 20 & 15 & 07 & 14 & 12 & 12 & 07 & 12 \\
\hline Intensidade da Concorrência & 10 & 16 & 09 & 12 & 14 & 12 & 07 & 12 \\
\hline $\begin{array}{l}\text { Investimento Equipamento e Tecnologia } \\
\text { (barreiras à entrada) }\end{array}$ & 15 & 08 & 07 & 07 & 07 & 03 & 03 & 04 \\
\hline Investimento RH (barreiras à entrada) & 10 & 07 & 03 & 07 & 06 & 03 & 03 & 07 \\
\hline Sinergias & 15 & 13 & 14 & 14 & 15 & 16 & 12 & 16 \\
\hline Possibilidade de Parceria externa & 10 & 12 & 12 & 04 & 12 & 12 & 12 & 14 \\
\hline $\begin{array}{l}\text { Potencial de ensino } \\
\text { Pesquisa }\end{array}$ & 20 & 17 & 17 & 14 & 15 & 15 & 16 & 16 \\
\hline Valor do segmento & 100 & 14,65 & 10,15 & 11,05 & 11,36 & 10,95 & 9,05 & 12,05 \\
\hline
\end{tabular}

$\mathrm{Na}$ matriz de análise do valor de cada segmento (tabela 1), observa-se que a possibilidade de crescimento foi considerada significativa nos segmentos UTI Neonatal e Alojamento Conjunto, devido à ampliação de leitos, que dobrou sua capacidade instalada de 10 para 20 leitos, e em função do perfil deste alojamento conjunto para atendimento de bebês de alto risco (recém-nascidos 
de baixo-peso e com malformação). Apesar da ampliação de leitos, de acordo com os atores envolvidos na aplicação da démarche, a maternidade ainda necessitaria de mais 46 leitos de UTI neonatal para atender à demanda. Ainda com relação à possibilidade de crescimento, os menores valores corresponderam a UCI Neonatal e Imunização, sinalizando uma tendência ao declínio. Com relação a este último segmento, discutiu-se que a vacinação é ofertada em todas as unidades básicas de saúde, mas a manutenção da demanda por este serviço da maternidade decorre da garantia de acesso durante todo o dia e pelo fato de os usuários receberem orientações e palestras. Registrou-se, por meio da ouvidoria da maternidade, que a confiança no serviço prestado também foi outro motivo da demanda dos usuários por esse serviço.

Levando-se em conta a quantidade e a capacidade de oferta de outras instituições, a intensidade de concorrência foi considerada baixa para os segmentos UTI Neonatal e Obstetrícia e média para os segmentos Pré-natal, Alojamento Conjunto e Apoio Diagnóstico, tendo em vista a diferenciação dos serviços prestados, o que implicou maiores notas. A maior quantidade de instituições e outros espaços da rede que não hospitais e que realizam atividades de imunização implicou a menor notação desse segmento neste critério.

Com relação ao nível do investimento a ser realizado em equipamentos ou tecnologias, capacidade instalada e insumos, tendo em vista um desempenho diferenciado (ou competitivo), foi atribuída uma nota alta para os segmentos UTI Neonatal, Apoio Diagnóstico, UCI Neonatal, Alojamento Conjunto e Obstetrícia; e nota média para os segmentos Pré-natal e Imunização. Porém, ao ser ponderado o nível do investimento já realizado e previsto para os próximos dois anos, os profissionais participantes concluíram que os investimentos já realizados em equipamento e tecnologias nos segmentos Apoio Diagnóstico, Prénatal e Imunização não constituem barreiras à entrada de novos investimentos, o que foi evidenciado por suas menores notações. Com relação aos segmentos UTI Neonatal, UCI Neonatal, Alojamento Conjunto e Obstetrícia, considerouse que, embora já tenha sido realizado grande investimento institucional em tecnologia e estrutura física, ainda há necessidade de maiores investimentos para que estes se tornem mais competitivos. 
A discussão do grupo traz à tona a questão da necessidade de investimentos em recursos humanos. $\mathrm{O}$ nível de investimentos necessários ou a ser realizado na capacitação dos recursos humanos e na melhoria eventual do quantitativo de pessoal foi considerado alto para os segmentos UTI Neonatal, Apoio Diagnóstico, Alojamento Conjunto e Obstetrícia; e médio para os segmentos UCI Neonatal, Pré-natal e Imunização. No entanto, ao se analisar o nível do investimento já realizado e o previsto para os próximos dois anos, o investimento necessário em uma competência específica ou num nível de capacitação, de aprendizagem ou de experiência particulares foi considerado médio para os segmentos UTI Neonatal, Apoio Diagnóstico e Alojamento Conjunto; baixo para o segmento Obstetrícia; e muito baixo para os segmentos UCI Neonatal, Pré-natal e Imunização.

A notação alta na avaliação das sinergias mostra o quanto os segmentos Prénatal, Apoio Diagnóstico, Obstetrícia, UCI Neonatal, Alojamento Conjunto e UTI Neonatal já trabalham de forma compartilhada. De forma geral, constatase que há integração e colaboração entre a Unidade Materno-Infantil e os outros serviços da maternidade. Ainda no item "Sinergia”, o segmento Imunização recebeu nota média, indicando ser mais fechado intra-institucionalmente. Apesar disso, a equipe profissional considera este serviço necessário, pois realiza a imunização de todos os recém-nascidos, tanto dos bebês internados quanto dos usuários externos.

O segmento com maior potencial de parceria externa é o Apoio Diagnóstico, expresso por alta notação, provavelmente em função de a demanda por esses serviços ser maior que a oferta. Porém, a possibilidade de parceria externa existe também para os segmentos: Obstetrícia, UTI Neonatal, UCI Neonatal, Pré-natal e Imunização, o que implicou notas médias. O Alojamento Conjunto apresenta a menor nota com relação a esse critério. Tal fato foi justificado pela inexistência de referências para este tipo de serviço na rede. Não há leitos disponíveis, nem tecnologia adequada para acolher estes usuários, tendo em vista que se trata de recém-nascidos que pesam entre 500 a $1.200 \mathrm{~g}$, muitos dos quais com problemas de má-formação, como problemas cardiológicos e gástricos.

Destaca-se o grande potencial de ensino e pesquisa em todos os segmentos da Unidade Materno-Infantil. 
Tabela 2. Posição Competitiva dos segmentos UTIN e UCI neonatal de uma maternidade pública estadual, de uma maternidade municipal e de uma maternidade privada. Teresina-PI, 2009

\begin{tabular}{|c|c|c|c|c|c|c|c|c|}
\hline \multirow{2}{*}{$\mathbf{N}^{\circ}$} & \multirow{2}{*}{$\begin{array}{l}\text { Fatores Chave de } \\
\text { Sucesso }\end{array}$} & \multicolumn{3}{|c|}{ UTIN MDER } & \multicolumn{2}{|c|}{$\begin{array}{c}\text { UTIN } \\
\text { Concorrente (1) }\end{array}$} & \multicolumn{2}{|c|}{$\begin{array}{c}\text { UTIN } \\
\text { Concorrente (2) }\end{array}$} \\
\hline & & Peso & Nota & $\begin{array}{c}\text { Score } \\
(=\operatorname{pxn} / 100)\end{array}$ & Nota & $\begin{array}{c}\text { Score } \\
(=\operatorname{pxn} / 100)\end{array}$ & Nota & $\begin{array}{c}\text { Score } \\
(=\operatorname{pxn} / 100)\end{array}$ \\
\hline 1 & $\begin{array}{l}\text { Competência } \\
\text { Profissional }\end{array}$ & 40 & 12 & 4,8 & 09 & 3,6 & 12 & 4,8 \\
\hline 2 & $\begin{array}{l}\text { Tecnologia Diagnóstica } \\
\text { e Terapêutica }\end{array}$ & 30 & 12 & 3,6 & 09 & 2,7 & 10 & 03 \\
\hline 3 & $\begin{array}{l}\text { Interaçáo da Equipe } \\
\text { Profissional }\end{array}$ & 30 & 12 & 3,6 & 09 & 2,7 & 11 & 3,3 \\
\hline \multirow{2}{*}{\multicolumn{2}{|c|}{$\begin{array}{l}\text { SCORE SOBRE O } \\
\text { SEGMENTO }\end{array}$}} & 100 & & 12,0 & & 9,0 & & 11,1 \\
\hline & & \multicolumn{3}{|c|}{ UCI NEO } & \multicolumn{2}{|c|}{ UCI } & \multicolumn{2}{|c|}{ UCI } \\
\hline 1 & $\begin{array}{l}\text { Competência } \\
\text { profissional }\end{array}$ & 30 & 12 & 3,6 & 09 & 2,7 & 12 & 3,6 \\
\hline 2 & Tecnologia diagnóstica & 30 & 12 & 3,6 & 09 & 2,7 & 10 & 03 \\
\hline 3 & $\begin{array}{l}\text { Interação da equipe } \\
\text { profissional }\end{array}$ & 10 & 13 & 1,3 & 10 & 01 & 12 & 1,2 \\
\hline 4 & Interação com a família & 15 & 12 & 1,8 & 09 & 1,35 & 12 & 1,8 \\
\hline 5 & Articulação com a rede & 15 & 12 & 1,8 & 09 & 1,35 & 10 & 1,5 \\
\hline \multicolumn{2}{|c|}{$\begin{array}{l}\text { SCORE SOBRE O } \\
\text { SEGMENTO }\end{array}$} & 100 & & 12,1 & & 9,1 & & 11,1 \\
\hline
\end{tabular}


Tabela 3. Posição Competitiva dos demais segmentos, da Unidade de Neonatologia de uma Maternidade Pública Estadual, de uma Maternidade Pública Municipal e de uma Maternidade Privada, Teresina-PI, 2009

\begin{tabular}{|c|c|c|c|c|c|c|c|c|}
\hline \multirow{2}{*}{ No } & \multirow{2}{*}{$\begin{array}{l}\text { Fatores Chaves de } \\
\text { Sucesso }\end{array}$} & \multicolumn{3}{|c|}{ Alojamento Conjunto MDER } & \multicolumn{2}{|c|}{$\begin{array}{c}\text { Alojamento Conjunto } \\
\text { USWF }\end{array}$} & \multicolumn{2}{|c|}{$\begin{array}{c}\text { Alojamento Conjunto } \\
\text { CSFÉ }\end{array}$} \\
\hline & & $\begin{array}{c}\text { Peso } \\
(\%)\end{array}$ & Nota & $\begin{array}{c}\text { Score }= \\
(\operatorname{pxn}) / 100\end{array}$ & Nota & $\begin{array}{c}\text { Score }= \\
(\mathrm{p} \times \mathrm{n}) / 100\end{array}$ & Nota & $\begin{array}{c}\text { Score }= \\
(\mathbf{p} \times \mathbf{n}) / \mathbf{1 0 0}\end{array}$ \\
\hline 1 & $\begin{array}{l}\text { Competência } \\
\text { Profissional }\end{array}$ & 25 & 12 & 3,0 & 09 & 2,25 & 12 & 03 \\
\hline 2 & $\begin{array}{l}\text { Tecnologia Diagnóstica } \\
\text { e Terapêutica }\end{array}$ & 25 & 12 & 3,0 & 09 & 2,25 & 10 & 2,5 \\
\hline 3 & $\begin{array}{l}\text { Interação Equipe } \\
\text { Profissional }\end{array}$ & 20 & 12 & 2,4 & 09 & 1,8 & 11 & 2,2 \\
\hline 4 & $\begin{array}{l}\text { Interação Equipe } \\
\text { Família }\end{array}$ & 10 & 12 & 1,2 & 09 & 0,9 & 12 & 1,2 \\
\hline 5 & Acompanhante & 10 & 12 & 1,2 & 09 & 0,9 & 12 & 1,2 \\
\hline 6 & Enfermaria canguru & 10 & 12 & 1,2 & 09 & 0,9 & 00 & 00 \\
\hline \multicolumn{2}{|c|}{$\begin{array}{l}\text { SCORE SOBRE O } \\
\text { SEGMENTO }\end{array}$} & 100 & & 12,0 & & 9,0 & & 10,1 \\
\hline & & \multicolumn{3}{|c|}{ Sala de Parto MDER } & \multicolumn{2}{|c|}{ Sala de Parto USWF } & \multicolumn{2}{|c|}{ Sala de Parto CSFÉ } \\
\hline 1 & $\begin{array}{l}\text { Competência } \\
\text { Profissional }\end{array}$ & 30 & 11 & 3,3 & 08 & 2,4 & 11 & 3,3 \\
\hline 2 & $\begin{array}{l}\text { Tecnologia Diagnóstica } \\
\text { e Terapêutica }\end{array}$ & 20 & 11 & 2,2 & 08 & 1,6 & 09 & 1,8 \\
\hline 3 & $\begin{array}{l}\text { Interação Equipe } \\
\text { Profissional }\end{array}$ & 15 & 12 & 1,8 & 09 & 1,35 & 11 & 1,65 \\
\hline 4 & $\begin{array}{l}\text { Interação Equipe } \\
\text { Família }\end{array}$ & 15 & 12 & 1,8 & 09 & 1,35 & 12 & 1,8 \\
\hline 5 & $\begin{array}{l}\text { Articulação com a } \\
\text { Rede }\end{array}$ & 10 & 13 & 1,3 & 10 & 01 & 11 & 1,1 \\
\hline 6 & Classificação de Risco & 10 & 12 & 1,2 & 09 & 0,9 & 10 & 01 \\
\hline \multicolumn{2}{|c|}{$\begin{array}{l}\text { SCORE SOBRE O } \\
\text { SEGMENTO }\end{array}$} & 100 & & 11,6 & & 8,6 & & 10,65 \\
\hline
\end{tabular}


Tabela 4. Posição Competitiva dos segmentos, da unidade materno-infantil de uma maternidade pública estadual, de uma maternidade pública municipal e uma maternidade privada segundo fatores estratégicos de êxito. Teresina-PI, 2009

\begin{tabular}{|c|c|c|c|c|c|c|c|c|}
\hline \multirow{2}{*}{ No } & \multirow{2}{*}{$\begin{array}{l}\text { Fatores Estratégicos } \\
\text { de Êxito }\end{array}$} & \multicolumn{3}{|c|}{ Pré-Natal MDER } & \multicolumn{2}{|c|}{$\begin{array}{c}\text { Pré-Natal } \\
\text { Concorrente (1) }\end{array}$} & \multicolumn{2}{|c|}{$\begin{array}{c}\text { Pré-Natal } \\
\text { Concorrente (2) }\end{array}$} \\
\hline & & Peso\% & Nota & $\begin{array}{c}\text { Score }= \\
(\mathbf{p x n}) / 100\end{array}$ & Nota & $\begin{array}{l}\text { Score }=(\text { pxn }) \\
\quad / 100\end{array}$ & Nota & $\begin{array}{c}\text { Score }=(\text { pxn }) \\
\quad / 100\end{array}$ \\
\hline 1 & Competência Profissional & 18 & 15 & 2,7 & 12 & 2,16 & 15 & 2,7 \\
\hline 2 & $\begin{array}{l}\text { Tecnologia Diagnóstica e } \\
\text { Terapêutica }\end{array}$ & 15 & 12 & 1,8 & 09 & 1,35 & 10 & 1,5 \\
\hline 3 & $\begin{array}{l}\text { Interação Equipe } \\
\text { Profissional }\end{array}$ & 7 & 7 & 0,49 & 04 & 0,28 & 06 & 0,42 \\
\hline 4 & $\begin{array}{l}\text { Interação Equipe } \\
\text { Profissional e Família }\end{array}$ & 15 & 12 & 1,8 & 09 & 1,35 & 12 & 1,8 \\
\hline 5 & Articulação com a Rede & 16 & 12 & 1,92 & 09 & 1,44 & 10 & 1,6 \\
\hline 6 & $\begin{array}{l}\text { Humanização do } \\
\text { Atendimento }\end{array}$ & 07 & 10 & 0,7 & 07 & 0,49 & 10 & 07 \\
\hline 7 & Curso para Gestantes & 15 & 12 & 1,8 & 09 & 1,35 & 00 & 00 \\
\hline 8 & $\begin{array}{l}\text { Atendimento } \\
\text { Multiprofissional para } \\
\text { Adolescentes e Gestantes } \\
\text { de Alto Risco }\end{array}$ & 07 & 10 & 0,7 & 07 & 0,49 & 10 & 0,7 \\
\hline \multicolumn{2}{|c|}{$\begin{array}{l}\text { SCORE SOBRE O } \\
\text { SEGMENTO }\end{array}$} & 100 & & 11,91 & & 8,91 & & 9,42 \\
\hline & & \multicolumn{3}{|c|}{ Imunização MDER } & $\begin{array}{c}\text { Imunização } \\
\text { C (1) }\end{array}$ & \multicolumn{3}{|c|}{$\begin{array}{l}\text { Imunização } \\
\mathrm{C}(2)\end{array}$} \\
\hline 1 & Competência Profissional & 30 & 14 & 4,2 & 11 & 3,3 & 14 & 4,2 \\
\hline 2 & $\begin{array}{l}\text { Tecnologia Diagnóstica e } \\
\text { Terapêutica }\end{array}$ & 35 & 15 & 5,25 & 12 & 4,2 & 13 & 4,55 \\
\hline 3 & $\begin{array}{l}\text { Interação da Equipe } \\
\text { Profissional }\end{array}$ & 20 & 10 & 02 & 07 & 1,4 & 09 & 1,8 \\
\hline 4 & Articulação com a Rede & 15 & 08 & 1,2 & 05 & 0,75 & 06 & 0,9 \\
\hline \multicolumn{2}{|c|}{$\begin{array}{l}\text { SCORE SOBRE O } \\
\text { SEGMENTO }\end{array}$} & 100 & & 12,65 & & 9,65 & & 11,45 \\
\hline & & \multicolumn{3}{|c|}{ Apoio Diagnóstico MDER } & Apoio Diag & \multicolumn{3}{|c|}{ Concorrente (2) } \\
\hline 1 & $\begin{array}{l}\text { Competência } \\
\text { Profissional }\end{array}$ & 22 & 13 & 2,86 & 10 & 2,2 & 13 & 2,86 \\
\hline 2 & Tecnologia diagnóstica & 20 & 12 & 2,4 & 09 & 1,8 & 10 & 02 \\
\hline 3 & $\begin{array}{l}\text { Interação com a Equipe } \\
\text { profissional }\end{array}$ & 13 & 07 & 0,91 & 04 & 0,52 & 06 & 0,78 \\
\hline 4 & Interação com a família & 20 & 12 & 2,4 & 09 & 1,8 & 12 & 2,4 \\
\hline 5 & Articulação com a rede & 25 & 12 & 03 & 09 & 2,25 & 10 & 2,5 \\
\hline \multicolumn{2}{|c|}{$\begin{array}{l}\text { SCORE SOBRE O } \\
\text { SEGMENTO }\end{array}$} & 100 & & 11,57 & & 8,57 & & 10,54 \\
\hline
\end{tabular}


Nas tabelas 2, 3 e 4, observa-se que foram estabelecidos como FCS para

todos os segmentos da Unidade Materno-Infantil, a competência profissional, a tecnologia diagnóstica/terapêutica e a interação entre a equipe profissional. A interação da equipe profissional com a família foi considerada fator-chave de sucesso para a maioria dos segmentos, mas não para os segmentos UTI Neonatal e Imunização. A articulação com a rede também foi considerada competitivamente relevante para quase todos os segmentos com exceção da UTI Neonatal e Alojamento Conjunto. Neste último, também foram valorizados como FCS a presença de acompanhante e o método Canguru. Além desses FCS, para a Obstetrícia foi considerada a classificação de risco e para o segmento Prénatal, a humanização, o curso de gestante e o atendimento multiprofissional para adolescentes e gestantes de alto risco.

Pode-se observar os maiores pesos atribuídos aos critérios mais ligados à tecnologia dura e leve-dura (MERHY, 1999), como a competência profissional e tecnologia diagnóstica/terapêutica em praticamente todos os segmentos, expressando, para o grupo de atores participantes, a melhor abordagem para enfrentar as situações complexas que cercam a saúde materno-infantil. Porém, também foi muito valorizado o critério "interação" entre a equipe profissional e articulação com a rede na maioria dos segmentos, expressando uma visão prospectiva capaz de projetar um novo modelo de atenção, com ênfase na equipe do tipo integração (PEDUZZI, 2001) e no fortalecimento de uma rede de cuidados. O grupo participante pareceu compreender que precisa necessariamente integrar-se para dar conta de responder às complexas demandas das mulheres e crianças.

O peso menor atribuído aos critérios reconhecidos como tecnologias leves ou relacionais (como interação da equipe profissional com a família, presença de acompanhante, método Canguru, classificação de risco, humanização, curso de gestante e atendimento multiprofissional para adolescentes e gestantes de alto risco) permite-nos identificar os pontos cruciais no processo de modificação e de reorganização do modelo de atenção institucional. Esse contexto nos remete ao surgimento de novas características sócio-culturais incoerentes com o padrão tradicional, mas ainda identificado como um quadro cultural contraditório, em 
transição (RIVERA, 2003). É possível aqui observar indícios do que Merhy (2000) chama de "transição tecnológica" e uma reestruturação produtiva do setor, ou seja, a existência de uma "ruptura tecnológica", uma vez que detectamos uma nova configuração tecnológica para a produção do cuidado em saúde, de tal modo que o objeto das ações de saúde e suas finalidades colocam-se como qualidades distintas das configurações anteriores.

O próximo passo executado foi a atribuição de notas de 0 a 20 para cada um dos critérios, considerando a avaliação atual sobre os FCS, que correspondem às competências/tecnologias que deve ser controlados para se obter êxito nas atividades em análise, considerando o controle da própria instituição e dos concorrentes/parceiros. Verificou-se uma dificuldade no que se refere à avaliação da concorrência. A identificação dos FCS apoia-se na observação do meio ambiente, na análise de um concorrente considerado ameaçador e na interrogação aos interessados, profissionais e usuários sobre os concorrentes ou possíveis parceiros para compor um cenário próximo da realidade. Essa identificação é indissociável da identificação dos concorrentes/parceiros e consiste em explorar, pela reflexão estratégica, certos dados interrogando-se sobre a persistência das expectativas observadas e sobre os fatores suscetíveis de modificação (ARTMANN, 2002). A análise foi feita em relação a duas maternidades, considerando a localização territorial e as características de proximidade em termos da assistência prestada.

A UTI Neonatal obteve uma notação média (entre 8 a 12) para todos FCS (competência profissional, a tecnologia diagnóstica/terapêutica e a interação entre a equipe profissional) da maternidade e dos concorrentes. E a UCI Neonatal obteve apenas no critério interação entre a equipe profissional uma nota alta. Para todos os outros FCS da maternidade e dos concorrentes, neste segmento, atribuíram-se notas médias.

O Alojamento Conjunto também obteve notas médias em praticamente todos os critérios da maternidade e dos dois concorrentes, com exceção da enfermaria Canguru no concorrente 2, que recebeu uma nota zero, por não ter o método implantado.

A Obstetrícia destacou-se pela obtenção de notas altas (entre 13 a 16) em quase todos os critérios, exceto na classificação de risco, na qual recebeu nota média, enquanto os dois concorrentes receberam notação média em todos os FCS. 
A nota referente à competência profissional foi alta no segmento Pré-natal tanto para a maternidade quanto para o concorrente 2 , e média para o concorrente 1. Contudo, tanto a maternidade quanto seus concorrentes contaram com notas baixas (entre 4 a 7) com relação à interação entre a equipe profissional. Além disso, no Pré-natal, o concorrente 1 também teve nota baixa atribuída aos critérios "humanização" e "atendimento multiprofissional para adolescentes e gestantes de alto risco" e o concorrente 2 teve nota muito baixa no item "curso de gestante".

Tanto nos segmentos Imunização quanto no Apoio e Diagnóstico, as notas também foram próximas com relação à maternidade e seus concorrentes, com leve vantagem para a maternidade estudada. Destaca-se a notação alta referente à competência profissional e à tecnologia diagnóstica/terapêutica na maternidade e concorrente 2 no segmento Imunização, e a nota alta com relação à competência profissional na maternidade e concorrente 2, no segmento Apoio e diagnóstico. Outra ressalva deve ser feita com relação à interação entre a equipe profissional, que teve notação baixa atribuída tanto para a maternidade quanto para os concorrentes no segmento Apoio e Diagnóstico, e também para o concorrente 1 no segmento Imunização.

Posteriormente, determinou-se a posição relativa da instituição em análise em face de seus concorrentes, por meio da comparação dos scores, resultado do produto dos pesos pelas notas dos FCS de cada segmento (tabelas 2, 3 e 4). De uma forma geral, em todos os segmentos a maternidade objeto do estudo encontra-se em melhor posição competitiva que os dois concorrentes; observou-se, no entanto, que em sua maioria a notação atribuída aos FCS da maternidade está muito próxima da notação do concorrente 2, com pequena vantagem com relação ao primeiro concorrente. As duas notas baixas que foram atribuídas à maternidade referem-se à interação entre a equipe profissional nos segmentos Pré-natal e Apoio Diagnóstico. Este item recebeu nota alta na UCI Neonatal e Obstetrícia. A pequena diferenciação positiva da maternidade com relação ao segundo concorrente foi conferida por meio de fatores relacionados à implementação da Política Nacional de Humanização na maternidade, tais como a Enfermaria Canguru e o curso para gestantes.

O portfólio (figura 1) foi construído a partir do valor, da posição competitiva e do volume de produção dos segmentos (quadro 1). 
Quadro 1. Dados para o portfólio da Maternidade Dona Evangelina Rosa - MDER

\begin{tabular}{|l|l|l|l|l|}
\hline $\mathrm{N}^{\circ}$ & Segmentos & Valor do Segmento & Posiçáo Competitiva & $\begin{array}{l}\text { Volume de produçáo do } \\
\text { Segmento (2009) }\end{array}$ \\
\hline 1 & UCI NEO & 10,15 & 12,1 & 1039 \\
\hline 2 & Alojamento Conjunto Alto Risco & 11,05 & 12 & 8682 \\
\hline 3 & UTI NEO & 14,65 & 12 & 453 \\
\hline 4 & Obstetrícia & 11,35 & 13,2 & 16368 \\
\hline 5 & Imunização IPS & 9,05 & 12,65 & 46847 \\
\hline 6 & Pré Natal IPS & 10,95 & 11,91 & 26600 \\
\hline 7 & Apoio Diagnóstico IPS & 12,05 & 11,57 & 127583 \\
\hline
\end{tabular}

Ele permite o diagnóstico do risco estratégico de cada segmento da instituição analisada e fornece uma ideia de conjunto e valor comparativo da posição concorrencial (ANDRADE; ARTMANN, 2009). A análise do portfólio demonstra a resultante da maior ou menor adaptação da oferta de serviços (valor da atividade) e do esforço requerido para chegar a excelência (posição competitiva).

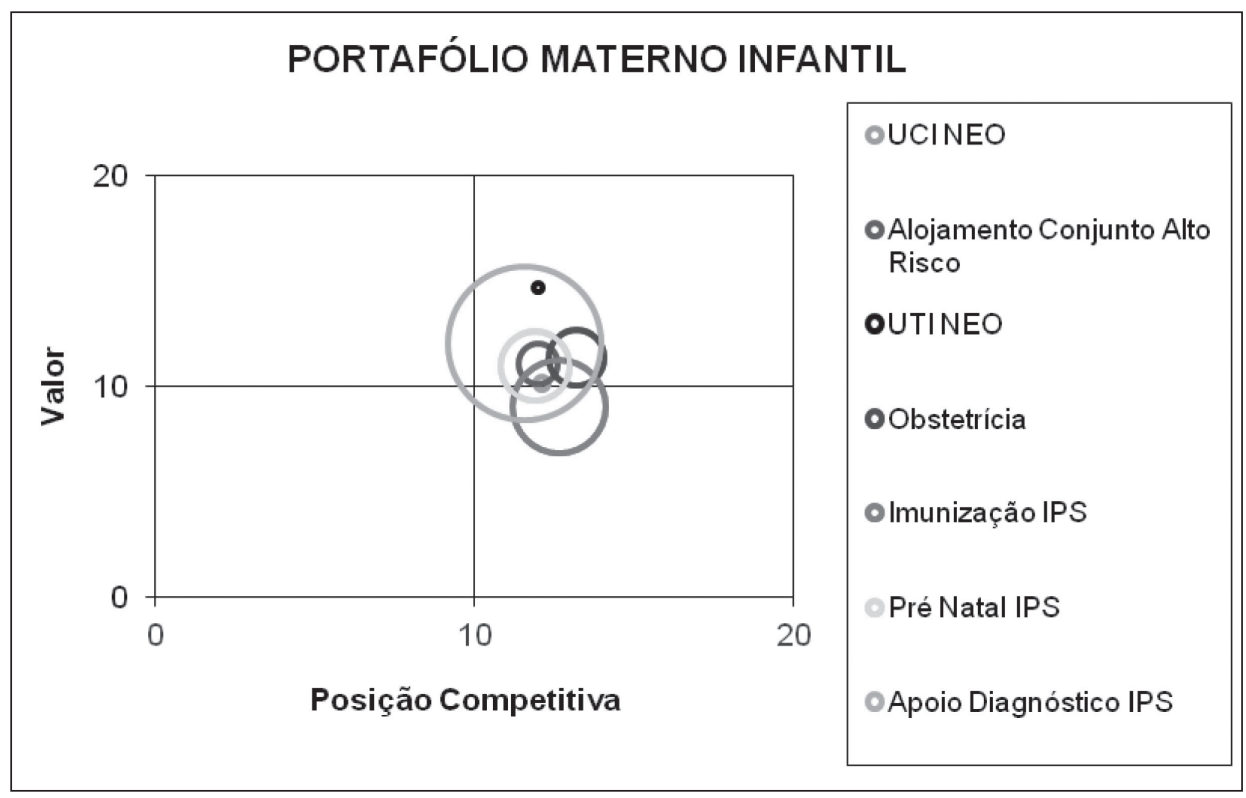


O portfólio divide-se em quatro quadrantes, sendo os dois superiores correspondentes a segmentos de alto valor, e os dois inferiores, a segmentos de baixo valor. Os quadrantes à esquerda representam os segmentos de baixa competitividade e os quadrantes à direita, os segmentos de alta posição competitiva. A análise do portfólio contribui para a tomada de decisão estratégica no plano de ação, especialmente focado em três possibilidades: 1) desenvolver um segmento, identificando as atividades que devem ser expandidas, priorizadas, desenvolvidas; 2) manter ou estabilizar um segmento, definindo os segmentos que devem ser mantidos, no mesmo nível de desenvolvimento, se possível reduzindo custos; 3) reduzir o recrutamento da clientela para um segmento, ou seja, definir quais segmentos seria necessário recortar ou focalizar, diminuir, negociar a transferência parcial ou total de atividades para a rede (ARTMANN, 2002).

Aqui é importante explicitar que o conceito de estratégico refere-se à busca de excelência no atendimento, levando em conta a demanda/necessidades da população, considerando que o estudo se refere a uma instituição pública e que o método foi idealizado justamente para o setor público. A comparação com outras instituições concorrentes tem o objetivo de identificar possíveis superposiçōes, mas também identificar atividades que podem ser oferecidas em parcerias. A ideia defendida é transformar concorrência em solidariedade para um ganho final do usuário.

O exame do portfólio demonstrou uma zona de indiferenciação ou de forte incerteza estratégica - situada na região central do gráfico - o que corresponde às atividades medianamente valorizadas e pouco discriminadas com relação à concorrência. Observa-se ainda, na estruturação do portfólio, uma tendência à direita, principalmente representada pelos Segmentos Obstetrícia e Imunização, indicando menor risco estratégico.

Com relação ao segmento Imunização, embora refletindo uma posição competitiva intermediária e uma grande produtividade, este não contribuiu para valorizar o projeto assistencial da maternidade, pois apresenta o menor valor dentre todos os segmentos. A estratégia de recorte por meio da definição da base territorial seria a mais adequada para este segmento, direcionando, se possível, parte da clientela para outras instituiçóes da rede.

O Apoio Diagnóstico é um segmento de grande volume de produção que incluiu os exames laboratoriais e de imagem, mas se encontra medianamente 
valorizado e com uma posição concorrencial desfavorável. Dada a impossibilidade de exclusão deste segmento, recomenda-se uma estratégia de focalização e diferenciação no sentido de assumir a realização de exames de maior complexidade, mais próximos da lógica hospitalar.

A UTI Neonatal apresenta-se como o segmento mais valorizado, mas com uma posição concorrencial também desfavorável. A estratégia sugerida é a diferenciação (ARTMANN; RIVERA, 2003), a partir de um investimento e de um conjunto de ações que aumentariam o valor e/ou a qualidade do atendimento.

Os segmentos UCI neonatal, Alojamento Conjunto e Pré-natal apresentam um valor e uma posição competitiva relativamente desfavoráveis. Para esses segmentos, a base da estratégia consistiria em enfrentar os pontos fracos da matriz dos FCS, de modo a avançar na diferenciação, em direção a um modelo mais focado no alto risco. O considerável volume de produção do segmento Pré-natal evidencia que grande parte da atividade não necessitaria ser realizada dentro da maternidade, podendo ser direcionada para outras unidades da rede. Esta medida levaria o segmento melhorar a competitividade e de qualidade, permitindo focalizar no acompanhamento de condições de saúde de maior risco.

A experiência permitiu uma observação direta e uma descrição detalhada sobre o ambiente interno, organizacional e a análise da produtividade, bem como sobre o ambiente externo, visto que foi considerada a rede regionalizada, os parceiros/concorrentes indicando prioridades para a construção de um plano de ação. Emergiram, na discussão do grupo, questões relacionadas ao fortalecimento de ações de humanização, como o Projeto Canguru e a instituição de um grupo de trabalho para repensar as práticas educativas e a educação permanente em Saúde-EPS na maternidade.

Considerando a rede como um modo de organização dos serviços sustentado por critérios, fluxos e mecanismos de pactuação, para assegurar a atenção integral aos usuários, evidenciou-se a necessidade de maior articulação com esta rede, bem como de um sistema de referência e contrarreferência mais efetivo para garantir a qualidade do atendimento na única maternidade de alta complexidade do estado. Nesse sentido, deve-se reafirmar a perspectiva de um desenho lógico de rede, que prevê níveis de complexidade, viabilizando encaminhamentos resolutivos (entre os diferentes serviços de saúde), porém reforçando sua concepção central de fomentar e assegurar vínculos em diferentes 
dimensões: intraequipes de saúde, interequipes/serviços, entre trabalhadores e gestores e entre usuários e serviços/equipes (BRASIL, 2010).

Essas questôes, junto com outras, como a necessidade de um sistema de informação padronizado e funcional, compuseram uma síntese propositiva que expressa os compromissos e ações para consecução das estratégias acima apresentadas e dos objetivos que visam a excelência no cumprimento da missão da maternidade (ARAÚJO, 2009)

\section{Consideraçōes finais}

A renovação do contrato entre os profissionais e a instituição depende da mobilização do papel da liderança, do papel da comunicação e da apropriação da organização pelos atores (RIVERA, 2003). A aplicação do método foi potente na apropriação da organização pelos atores, ao discutir a organização dos processos de trabalho hospitalar, numa perspectiva de rede coordenada de serviços no território onde se encontra inserida a maternidade.

A valorização de fatores-chave referentes às tecnologias relacionais e ao fortalecimento da rede sinaliza um entendimento de que apenas maior acesso à tecnologia dura, aos insumos e equipamentos biomédicos não assegura por si a reversão desse quadro. Há necessidade de um olhar mais ampliado do processo saúde/doença e de uma prática de cuidado mais integral. Destaca-se, no entanto, que no segmento UTI Neonatal, nem as tecnologias leves nem a constituição de redes ainda foram suficientemente valorizadas como fatores de sucesso. Contudo, uma mudança cultural mais profunda exige maior tempo histórico para se efetivar.

Esta experiência de aplicação da démarche permite afirmar uma concepção inovadora e efetiva em produzir equipes de saúde mais solidárias e corresponsáveis com movimentos de mudança das práticas. Foram consideradas várias dimensões de análise organizacional e de produtividade, permitindo a criação de canais de comunicação entre os profissionais da maternidade e entre estes com atores da rede externa de serviços. O método incluiu ainda os diferentes atores nas discussões sobre os processos de trabalho e na avaliação dos resultados e impactos na produção organizacional. Ressalta-se a importância da reflexão cotidiana sobre os processos de trabalho e na necessidade de empoderamento e autonomia dos profissionais de saúde na busca pela implementação de um modelo de atenção e gestão mais humanizada e de construção do processo democrático no SUS. 
Os resultados apresentados mostram ainda a capacidade do método para problematizar os processos de trabalho e para a análise da gestão hospitalar, apontando estratégia para melhoria da qualidade do cuidado e para maior inserção e integração na rede de saúde. Por apresentar critérios definidos, o método permitiu análises e comparações mediante a organização de informações e possibilitou validações, observaçōes e visões dos diferentes atores envolvidos no processo, levando-se em consideração suas experiências e especificidades. A diminuição da subjetividade pode ser alcançada em um contexto de participação suficientemente amplo para possibilitar o consenso (ARTMANN; RIVERA, 2003).

$\mathrm{O}$ estudo mostrou a potencialidade do enfoque como um instrumento de diagnóstico situacional e de ferramenta de gestão hospitalar para rever a missão institucional na busca de qualidade e solidariedade no interior da rede e no compromisso com o atendimento ao usuário mais universal e equânime. Contudo, os compromissos expressos na síntese propositiva devem ser constantemente revistos e repactuados entre os atores, para deixarem de ser uma carta de intenções e se constituírem em um plano de ação concreto, com o fim de alcançar mudanças institucionais permanentes. ${ }^{1}$

\section{Referências}

ANDRADE, M.A.C.; ARTMANN, E. Démarche estratégica em unidade materno infantil hospitalar. Rev. Saúde Pública. São Paulo, v. 43, n. 1, p. 105-14, 2009.

ARAÚJO, M.J.D. Démarche Estratégica em Unidade Materno-Infantil do Piauí: análise da participação na gestão do processo de humanização. Dissertação (Mestrado profissional) Escola Nacional de Saúde Pública, Fiocruz, Rio de Janeiro, 2009.

ARTMANN, E.; RIVERA, F.J.U. A démarche stratégique (gestáo estratégica hospitalar): um instrumento de coordenação da prática hospitalar baseado nos custos de oportunidade e na solidariedade. Ciênc. saúde coletiva. Rio de Janeiro, v. 8, n. 2, p. 479-499, 2003.

ARTMANN, E. Enfoque da démarche stratégique na gestão hospitalar. In: MINAYO, M.C.S.; DESLANDES, S.F. (Orgs.). Caminhos do pensamento: epistemologia e método. Rio de Janeiro: Fiocruz; 2002. p. 277-323.

BENEVIDES, R.; PASSOS, E. A humanização como dimensão pública das políticas de saúde. Revista Ciênc. saúde coletiva. Rio de Janeiro, v. 10, n. 3, p. 561-571, 2005.

BRASIL. Ministério da Saúde. Secretaria de Atenção à Saúde. Núcleo Técnico da Política Nacional de Humanização. HumanizaSUS: Documento base para gestores e trabalhadores do SUS. 4. ed. 4. reimp. Brasília: Ministério da Saúde, 2010. 
BRASIL. Ministério da Saúde. SecretariaExecutiva. DepartamentodeApoioà Descentralização.

Coordenação-Geral de Apoio à Gestão Descentralizada. Diretrizes operacionais dos pactos pela vida, em defesa do SUS e de gestão. Brasília: Ministério da Saúde, v. 1, 2006.

CECÍLIO, L.C. de O. A modernização gerencial dos hospitais públicos: o difícil exercício da mudança. Revista de Administração Pública, Rio de Janeiro, v. 31, n. 3, p. 36-47, 1997.

CRÉMADEZ, M.; GRATEAU, F. Le management stratégique hospitalier. Paris: InterÉditions, 1997. 448 p.

DAVIS-FLOYD, R. et al. A iniciativa internacional pelo nascimento mãebebê: uma abordagem de um atendimento materno eficiente à luz dos direitos humanos. Rev Tempus Actas Saúde Col. Brasília, v. 4, n. 4, 2010.

DESLANDES, S.F. Humanização: revisitando o conceito a partir das contribuições da sociedade médica. In: (Org.). Humanização dos cuidados em saúde: conceitos, dilemas e práticas. Rio de Janeiro: Fiocruz, 2006. p. 205-231.

DINIZ, S.G. Gênero, saúde materna e o paradoxo perinatal. Rev. bras. crescimento desenvolv. hum., São Paulo, v. 19, n. 2, p. 313-326, ago. 2009.

MERHY, E.E. O ato de governar as tensões constitutivas do agir em saúde como desafio permanente de algumas estratégias gerenciais. Ciênc. saúde coletiva. Rio de Janeiro, v. 4, n. 2, p. 305-314, 1999.

- Um ensaio sobre o médico e suas valises tecnológicas: contribuições para compreender as reestruturações produtivas do setor saúde. Interface (Botucatu), Botucatu, v. 4, n. 6, p. 109-116, fev. 2000.

PASCHE, D.F.; VILELA, M.E. de A.; MARTINS, C.P. Humanização da atenção ao parto e nascimento no Brasil: pressuposto para uma nova ética na gestão e no cuidado. Rev Tempus Actas Saúde Col, Brasília, v. 4, n. 4, p. 105-117,2010.

PEDUZZI, M. Equipe multiprofissional de saúde: conceito e tipologia. Rev. Saúde Pública, São Paulo, v. 35, n. 1, p. 103-109, fev. 2001.

RIVERA, F.J.U. A démarche estratégica: a metodologia de gestão do Centro Hospitalar Regional Universitário de Lille, França. Cad. Saúde Pública, Rio de Janeiro, v. 13, n. 1, p. 73-80, jan. 1997.

RIVERA, F.J.U. Cultura e Liderança Comunicativa. In: Análise Estratégica em saúde e Gestão pela Escuta. Rio de Janeiro: Fiocruz, 2003, p. 37-96.

SESAPI. Secretaria de Saúde do Estado do Piauí. Disponível em: http://sesapi.pi.gov.br acesso em 18 mar 2009.

VICTORA, C.G. et al. Saúde de mães e crianças no Brasil: progressos e desafios. Disponível em: http://download.thelancet.com/flatcontentassets/pdfs/brazil/brazilpor2.pdf. Acesso em: 07 nov. 2011. 


\section{Nota}

${ }^{1}$ M.J.D. Araújo realizou a coleta e interpretação de dados; redação do artigo e revisão e aprovação final. E. Artmann participou da concepção do projeto; interpretação dos dados; redação e revisão e aprovação final do artigo. M.A.C. Andrade realizou a interpretação dos dados e redação e aprovação final do artigo. 
Strategic Démarche: innovative and effective way to analyze the institutional mission In Brazil, despite the improvements that have occurred in the health of mothers and children, indicators of maternal and neonatal mortality are still far from desirable. To reverse this situation, it is important to rethink the process of work, considering the hospital and perinatal care network planning, and focus on institutional democratization. This study aimed to discuss the process of application of the "strategic demarche" approach in a State public maternity with a view to coordinated network of services. The period was from June to September 2009 and included the direct participation of professionals / stakeholders, including the hospital board. The results showed the method's ability to question the work processes and the analysis of hospital management. Based on the analyses, the experience pointed action strategies validated by different actors. The method can be considered innovative and effective to produce more cohesive health teams and co-responsible with changing practices movements. It allowed the creation of communication channels between maternity professionals, and between these actors and the external network. The study showed the potential of the approach as a tool for situational analysis and hospital management to review the institutional mission in search of quality and solidarity within the network, committed to attend users in a universal and equitable way. However, the commitments expressed in the propositional synthesis must be constantly reviewed and renegotiated by the actors, in order to achieve permanent institutional changes.

Key words: Public Health; strategic management; health planning; humanization; child and mother health care. 